Ciencia y Educación, Vol. 4, No. 2, mayo-agosto, 2020

ISSN (impreso): 2613-8794・ISSN (en línea): 2613-8808

DOI: https://doi.org/10.22206/cyed.2020.v4i2.pp53-66

\title{
Una mirada al diseńo instruccional y al desarrollo profesional de docentes de inglés como lengua extranjera
}

\author{
A look at instructional design and professional development \\ of english teachers as a foreign language
}

Alexander López Díaz ${ }^{a}$ ORCID: 0000-0002-5995-5042

Recibido: 21/12/2019• Aprobado: 26/01/2020

Cómo citar: López Díaz, A. (2020). Una mirada al diseño instruccional y al desarrollo profesional de docentes de inglés como lengua extranjera. Ciencia y Educación, 4(2), 53-66. Doi: https://doi.org/10.22206/cyed.2020.v4i2.pp53-66

\section{Resumen}

La enseñanza del Inglés como Lengua Extranjera (ILE) es un área de amplio crecimiento en República Dominicana. En este contexto, la figura del docente de ILE es preponderante. Este artículo tiene el objetivo de identificar dos aspectos clave en el campo profesional de la enseńanza: Diseño Instruccional (DI) y Desarrollo Profesional Docente (DPD). En primer lugar, se analiza la percepción de estos docentes concerniente a la secuencia o estructura del proceso de enseñanza-aprendizaje. En segundo lugar, se estudia qué consideran los maestros como DPD. Para recolectar datos relevantes, fue utilizada la encuesta como instrumento de investigación a través de un formulario de Google Forms $(n=79)$. Los resultados de este estudio plantean una presencia predominante de enfoques tradicionales en la enseñanza del ILE que no refleja la corriente posmétodo actual, y una idea turbia con respecto a lo que realmente supone el desarrollo profesional docente, altamente limitada a programas de formación y promoción laboral. Al final del estudio, se discuten las implicaciones de estos resultados y recomendaciones para docentes en servicio.

Palabras clave: enseñanza; desarrollo de la carrera; enseñanza de una lengua extranjera; instrucción.

\begin{abstract}
Teaching English as a Foreign Language (EFL) is a rapidly growing area in the Dominican Republic. In this context, the figure of the EFL teacher is predominant. This article aims to identify two key aspects in the professional field of teaching: Instructional Design (ID) and Teacher Professional Development (TPD). Firstly, the perception of these teachers regarding the sequence or structure of the teaching-learning process is analyzed. Second, we study what teachers consider to be TPD. To collect relevant data, the survey was used as a research instrument through a Google Forms form $(n=79)$. The results of this study suggest a predominant presence of traditional approaches in the teaching of EFL that does not reflect the current post-method current, and a murky idea regarding what professional teaching development really means, highly limited to training and job promotion programs. At the end of the study, the implications of these results and recommendations for in-service teachers are discussed.
\end{abstract}

Keywords: teaching; career development; teaching a foreign language; instruction.

a Concordia University Chicago, Estados Unidos. Correo-e: crf_lopezda2@chicago.edu 


\section{Introducción}

La enseñanza de inglés es un paradigma de interés para el mundo global en que vivimos. El aprender un nuevo idioma se enmarca en las metas de profesionales alrededor del mundo, quienes, debido a diversas razones y motivaciones, deciden embarcarse en la misión de aprender una nueva lengua. Dicho crecimiento de la enseñanza y el aprendizaje de lenguas extranjeras no es externo a la realidad dominicana. República Dominicana ha visto un vertiginoso avance en esta área, y numerosas instituciones han sido creadas con el propósito de brindar esta oportunidad a la ciudadanía. Se precisan docentes de calidad y con alto dominio de la lengua para contribuir de manera significativa en este esfuerzo. En el país, por ejemplo, la diversificación de programas e instituciones que tienen como meta la enseñanza de idiomas, hace que más docentes se interesen por este campo con miras a contribuir al avance socioeconómico de la nación. Junto con esta realidad, el docente de lenguas extranjeras se debe perfilar como un profesional informado, crítico y altamente capacitado.

Sin embargo, a pesar del crecimiento del bilingüismo y la enseñanza de ILE en el país, pocos trabajos investigativos permiten estudiar la realidad y estado de este campo educativo. Es necesario disponer de conocimiento válido y confiable que explique las tendencias y defina de manera más clara el perfil del docente de ILE en el país. El presente estudio supone una contribución original en este aspecto. Específicamente, este artículo examina las percepciones de maestros en servicio concernientes al diseño instruccional y el desarrollo docente o desarrollo profesional. Este estudio utiliza la encuesta como instrumento de investigación para responder a las interrogantes: ¿qué consideran los maestros dominicanos de Inglés como desarrollo profesional? y ¿Qué diseño instruccional siguen los maestros de Inglés en sus lecciones? Para contextualizar la discusión de dichas preguntas, se recorren los diferentes estudios de autores que han investigado temas similares o son expertos en el campo. Luego, se presenta la articulación metodológica utilizada seguida de los resultados. Para finalizar, se analizan dichos resultados a la luz de la literatura consultada y la realidad de la enseñanza de idiomas dentro del contexto dominicano.

\section{Revisión de la literatura}

\subsection{Diseño Instruccional (DI)}

El diseño instruccional (DI) se presenta como un estudio de los principios relacionados con la enseñanza y aprendizaje en el aula. Jardines Garza (2011) establece que el "DI es un proceso complejo que, cuando es aplicado de manera apropiada, promueve creatividad durante el desarrollo y resultados en la instrucción que lo hace eficaz y atractivo a los estudiantes" (p. 360). Dicha definición supone que al hablar de DI, el profesional de la educación se refiere a procesos enmarcados en el desarrollo y resultados de la instrucción. En esta disciplina, el término instrucción es definido por Luengo Navas (2004) como "un proceso de enseñanza en el que el educador elige determinados contenidos educativos para que el sujeto los aprenda con una escasa participación de la comprensión" (p. 42).

Gagné, Briggs y Wager (1992) enmarcan el estudio del DI con la pregunta: ¿cómo se debe organizar la instrucción? Para estos autores, el estudio del DI yace en la idea de que la enseñanza organizada sistemáticamente puede afectar de manera significativa el desarrollo humano individual (Gagné et al.,1992, p. 5). Otros investigadores también aportaron al desarrollo de la conceptualización y operacionalización del DI. Parra y Leyet (2012) proveen una enriquecedora discusión acerca de los modelos de estructuración más comunes, dentro de los cuales se mencionan Dick y Carry (1978), quienes sugerían la especificación de objetivos y la culminación de la experiencia con una evaluación sumativa; Gerlach y Ely (1980) con un sistema enfocado en pasos; Merrill (1983), quien clasificaba la experiencia de aprendizaje en contenido y comportamientos; Heinich, Molenda, Russell y Smaldino (1999), quienes toman en cuenta al estudiante en el desarrollo del DI; culminando con Kemp (1985), que también se centra en el estudiante y sus necesidades, e incorpora la evaluación sumativa $\mathrm{y}$ formativa.

En este artículo, el DI se define como el estudio y la sistematización de las diversas etapas propias del proceso de enseñanza-aprendizaje. Dicha definición se relaciona con las premisas básicas de Gagné et al., (1992), que plantean procesos, sistematicidad, y pasos. 
Es importante tener presente que las diferentes escuelas filosóficas dentro de la educación y los enfoques metodológicos intervienen directamente en la instrucción. En todo caso de estudio del DI, la figura del docente es resaltada debido a su papel crucial en la estructuración de la experiencia de aprendizaje. Es parte del rol del educador analizar las necesidades, el perfil y el entorno de los estudiantes y utilizar esta información para proveer una solución educativa relevante para el estudiantado. Es decir, es parte del trabajo del maestro ajustar a las características del estudiantado los diversos pasos que seguirá cierta lección o programa. A medida que el docente es un profesional crítico e informado, las decisiones concernientes al DI pueden ser más efectivas y atinadas con la realidad en cuestión.

\subsection{Enseñanza de Inglés como Lengua Extranjera (ILE) y Diseño Instruccional (ILE)}

La enseñanza del idioma inglés ha sido protagonista en el desarrollo de teorías de enseñanza-aprendizaje e innovación metodológica. En el desarrollo de esta disciplina, se visualizaba una gran influencia conductista apoyada por la lingüística estructuralista. Sin embargo, más recientemente, dentro del enfoque constructivista, la enseñanza de inglés es concebida como un instrumento útil para la comunicación entre individuos y grupos de diversas culturas. Bajo esta perspectiva, la enseńanza de lenguas extranjeras sirve a un propósito comunicativo. El método comunicativo que nació en los años ochenta, del siglo xx, tiene como visión la práctica relevante y auténtica que prepare al estudiante para utilizar la segunda lengua en un contexto real (Richards y Rodgers, 2016). La repetición y la memorización pierden autoridad bajo este enfoque. Como partida, se toma el concepto de Hymes (1972) con respecto a la competencia comunicativa; de ahí se trabajan funciones o nociones. Mato (2011) observa cómo esta perspectiva "intenta exponer una forma natural del lenguaje, basado en la comunicación diaria" (p. 16). Propio del enfoque comunicativo son las conversaciones en el aula, los diálogos en pareja, los juegos didácticos, el uso de actividades orientadas al aprendizaje y la práctica auténtica del idioma en el contexto del aula. Los estudiantes son motivados a usar la lengua y desarrollar fluidez, aparte de precisión gramatical.

Sin embargo, mientras muchos consideran que el método comunicativo es el actual, y que este es representativo de las tendencias didácticas del momento, otros, como Kumaravadivelu (2003), abogan por un entendimiento posmétodo, en el cual los principios comunes en la enseńanza de lenguas extranjeras se sobrepongan a los limitados y prescriptivos métodos establecidos. Para esta autoridad en el campo, la palabra método ha perdido valor debido a su subjetividad y a su naturaleza limitante. Este considera que una perspectiva posmétodo es en la que vivimos actualmente, y que esta se sobrepone a los métodos anteriores ya que: (1) significa una alternativa a los métodos, no un método alternativo; (2) promueve la autonomía del docente; y (3) está basado en el pragmatismo (Kumaravadivelu, 2003, pp. 32-34).

En general, un análisis crítico a la evolución de la metodología en la enseñanza de inglés, evidencia cambios significativos en el DI de ILE. Una comparación de los principios expuestos en cada método puede esclarecer pasos en su DI. Las diversas estrategias utilizadas en el aula constituyen en sí mismas una representación de la visión del momento con respecto a la enseñanza de lenguas extranjeras, y su secuenciación está basada en las innovaciones de la investigación. Es por esto que la palabra método, dentro de este campo, está íntimamente ligado a estrategias dentro del DI que, en muchos casos, están basadas en conceptos o ideales específicos. De hecho, es común ver cómo las clases de lenguas extranjeras adoptan el DI propuesto por el método asumido por el docente o la institución a la que este pertenece. En la enseñanza de ILE, más allá de los diversos enfoques, Richards y Lockart (1996) proponen un DI (al que se refieren como estructuración) compuesto por cuatro fases:

1. Apertura: cómo se inicia la lección.

2. Secuenciación: cómo se divide la lección entre segmentos y cómo estos se relacionan entre sí.

3. Ritmo: cómo se alcanza un sentido de progreso en una lección.

4. Cierre: cómo se concluye con una lección. 
Para estos autores, cada uno de estos segmentos en la lección tiene principios que aseguran su efectividad y que guían la selección de actividades y materiales para cada uno.

\subsection{La enseñanza de DI en República Dominicana}

En el país, la enseñanza de lenguas extranjeras ha ganado atención en los últimos años. Actualmente, existe la demanda de ciudadanos bilingües para contribuir al avance socioeconómico y la diversificación de la nación. Un área de reciente interés es la industria de zonas francas especiales, o call centers, que requieren de empleados con alta competencia comunicativa en inglés. En retrospectiva, República Dominicana tiene larga historia en la enseñanza del idioma inglés. Una de las más antiguas instituciones dedicadas a la enseñanza de ILE, es el Instituto Cultural Domínico Americano (ICDA), fundado en 1947 por un grupo de norteamericanos. En el sector privado, este instituto es considerado un pionero. Sin embargo, el ICDA no es el único organismo proveedor de este servicio. El sector privado ha exhibido vertiginoso crecimiento, visto a través del establecimiento de varios programas e instituciones de enseñanza de lenguas extranjeras, muchos con capital humano y métodos innovadores foráneos.

En el sector público, el Estado Dominicano ha enmarcado el Programa de Inglés de Inmersión, desarrollado por el Ministerio de Educación Superior, Ciencia y Tecnología (MESCyT). Este programa, destinado a estudiantes universitarios, atrae anualmente a miles de jóvenes de diversos estratos sociales a participar en clases de inglés, por cuatro horas diarias, de lunes a viernes. Dicho programa tiene el objetivo de llevar a los estudiantes al nivel B2 (usuario independiente) en el Marco Común Europeo de Referencias para las Lenguas (MCERL). Dicho programa requiere que los estudiantes y maestros utilicen el inglés como único medio de instrucción, lo cual supone un reto para los alumnos (López, 2019). Por otro lado, en la educación preuniversitaria, actualmente inmersa en reforma curricular, la enseñanza de ILE comienza en el Segundo Ciclo del Nivel Primario, de acuerdo al currículo dominicano, específicamente en el cuarto grado y hasta finalizar la secundaria.

Actualmente, se dispone de pocos estudios publicados que retraten la realidad de la enseñanza de ILE en la nación. De los trabajos disponibles, la mayoría corresponden a tesis de grado o posgrado. En resumen, dichos estudios están de acuerdo con el uso de métodos tradicionales de enseñanza que no necesariamente fomentan la discusión y el uso fluido de la lengua objeto en el aula (Bonilla-Reynoso, 2014; Murray Sosa y Suero, 2015; López, Martínez, Pérez, Jiménez y Mateo 2019). Un factor al que no escapa la enseńanza de ILE en el país, es la sobrepoblación de estudiantes en las aulas. Santana (2016) expone cómo este fenómeno impacta negativamente al aprendizaje. En general, el panorama de la enseńanza de lenguas extranjeras en el país apunta al uso de estrategias tradicionales, propias de enfoques de enseńanza que ya han sido desplazados por innovaciones en el área que prestan atención al desarrollo de la competencia comunicativa, como el enfoque comunicativo, ampliamente discutido en este campo educativo (Larsen-Freeman y Anderson, 2011; Richards y Rodgers, 2016).

\subsection{Desarrollo profesional docente}

El docente de lenguas extranjeras es igualmente una figura central en la literatura. Este docente es considerado como un profesional que, aparte de tener una evidente capacidad y manejo lingüístico de la lengua que enseña, tiene un amplio conocimiento metodológico que fomente el aprendizaje en el aula. El docente de lenguas extranjeras tiene una misión específica dentro de la educación. Fandińo-Parra (2017) expone que "la meta del docente de lenguas es ayudar a que los estudiantes y los individuos se conviertan en usuarios eficientes, creativos y críticos" (p. 124). Se observa cómo la efectividad de este profesional está basada en el desarrollo de las habilidades lingüísticas de sus estudiantes. Para lograr esta meta, la formación docente juega un papel fundamental.

De acuerdo a Villegas-Reimers (2002), citado por Fandiño-Parra (2017), la formación docente es 
un proceso de larga duración basado en experiencias programadas para fomentar el crecimiento y el desarrollo en la profesión (p. 126). Por su parte, los programas de formación de docentes de Lenguas Extranjeras tienen como objetivo la reflexión profesional y la ejecución casi automáticas de técnicas y estrategias en el aula (Fandińo-Parra, 2017). Por otro lado, el desarrollo docente es sinónimo del crecimiento profesional producto de la experiencia y la reflexión profesional propia. Es decir, va más allá de los programas de formación y se evidencia en la práctica docente misma, es activo, y se enfoca en el autodescubrimiento del docente (Whitehead, 1989; Fandiño, 2010; Fandiño-Parra, 2017). Para Richards y Farell (2005) el desarrollo en docentes de inglés tiene las siguientes metas:

- Entender cómo los procesos de una segunda lengua ocurren.

- Entender cómo nuestros roles cambian de acuerdo al tipo de estudiante que educamos.

- Entender las formas de toma de decisiones que ocurren dentro de una lección.

- Revisar nuestras propias teorías y principios en la enseńanza de idiomas.

- Desarrollar un entendimiento de los diferentes estilos de enseñanza.

- Determinar la percepción del estudiante acerca de las actividades del aula. (p. 4)

Para estos autores, la discusión acerca de desarrollo en docentes de lenguas extranjeras no puede dejar atrás la distinción entre docentes noveles y expertos. Las diferencias entre los mismos se explican como una base de conocimiento más rica y elaborada, habilidad para integrar y usar diferentes tipos de conocimientos, habilidad para hacer juicios correctos e intuitivos basados en experiencia previa, un deseo de investigar y resolver diferentes problemas de enseñanza; entre otros (Richards y Farrell, 2005, p. 8). El crecimiento, desde novel hasta experto, es notable en la implementación de actividades y la anticipación de problemas instruccionales y su posible solución (Berliner, 1987, citado por Richards y Farrell, 2005). El desarrollo docente tiene dos paradigmas a tomar en cuenta: los objetivos institucionales y las metas individuales del docente (Richards y Farrell, 2005, p. 9). La tabla a continuación explica ambos.
Tabla 1. Paradigmas para el desarrollo docente

\begin{tabular}{|l|l|}
\hline Perspectiva individual & Perspectiva institucional \\
\hline Conocimiento de la materia & Desarrollo institucional \\
\hline Pericia pedagógica & $\begin{array}{l}\text { Desarrollo dentro de la } \\
\text { carrera }\end{array}$ \\
\hline Autoconocimiento & $\begin{array}{l}\text { Niveles mejorados de } \\
\text { aprendizaje en el alumnado }\end{array}$ \\
\hline $\begin{array}{l}\text { Conocimiento de los } \\
\text { estudiantes }\end{array}$ & $\begin{array}{l}\text { Conocimiento del currículo y } \\
\text { los materiales }\end{array}$ \\
\hline Avance dentro de su carrera & \\
\hline
\end{tabular}

Fuente: elaboración propia.

La actual reforma curricular y los avances en el área educativa han prestado atención especial a la formación y desarrollo docente. En un artículo acerca de las políticas docentes dominicanas, Van Grieken (2018) explica las últimas regulaciones establecidas por el Ministerio de Educación de la República Dominicana (MINERD), las cuales constituyen las Dimensiones del Profesionalismo y Desempeño Docente, y suponen cuatro estadios: (1) el estudiante, la estudiante y su aprendizaje, (2) contenido curricular, (3) proceso de aprendizaje-enseńanza, y (4) compromiso personal y profesional. Estas políticas rigen la formación docente en el país.

En el área de ILE, nacionalmente, los programas de grado y posgrado constituyen la mayor fuente de formación profesional para los maestros de inglés. Dentro de los programas de grado podemos contar con las licenciaturas en Lenguas Modernas, ofertadas por la Universidades Autónoma de Santo Domingo (UASD) y la Universidad Tecnológica de Santiago (UTESA), entre otras; además de Inglés Orientado a la Enseñanza, de la Universidad del Instituto Cultural Dominico Americano (UNICDA), recientemente se le unió la Universidad Acción Pro Educación y Cultura (UNAPEC), con una licenciatura en Lenguas Extranjeras. En el área del posgrado, los programas son pocos. La UASD, por su lado, prepara la mayor cantidad de profesionales con su maestría en Lingüística Aplicada a la Enseñanza del ILE, mientras 
que UNICDA y la Pontificia Universidad Católica Madre y Maestra (PUCMM), ofrecen maestrías en Enseñanza de ILE. En el área de Educación Continuada, se conoce de los programas de certificación avalada por la School of International Training (SIT) en TESOL (Enseñanza de Inglés para hablantes de otras lenguas, por sus siglas en inglés), provistos en conjunto con la Embajada de los Estados Unidos. y el Centro Espiral Maná, aparte del Teaching Knowledge Test de Cambridge English Language Assessment, manejado por la Escuela de Idiomas de UNAPEC. Grosso modo, esos son los principales programas de formación al cual tienen acceso los maestros de ILE dominicanos, aparte de las conferencias organizadas por TESOL República Dominicana, el Instituto Cultural Domínico Americano y la Embajada Americana en el país.

\section{Metodología}

Este estudio tiene como principal objetivo analizar el DI de los maestros de inglés en sus lecciones e identificar las percepciones de los mismos con respecto al desarrollo docente. La encuesta es tomada como instrumento de investigación. López-Roldán y Facheli (2015) destacan cómo la encuesta ha llegado a ser fundamental en el campo de la investigación social, al punto de considerarse en sí misma un método. Estos autores ponderan cómo el uso de la encuesta en la investigación científica añade objetividad al estudio en general. Este estudio considera adecuada la aplicación de la encuesta para la recolección de datos, debido a que sus resultados ayudan a entender la percepción de docentes respecto a los dos fenómenos anteriormente descritos, de una forma más directa. La encuesta, como instrumento, añade objetividad al estudio, representada de forma que permite captar de primera mano las experiencias y percepciones del encuestado. Además, la selección de la encuesta como instrumento de investigación tuvo que ver con la facilidad de alcance que esta provee. Se utilizó un formulario online, en inglés, distribuido a maestros de diversas instituciones y localidades dentro del país. La cantidad de los participantes corresponde a una muestra aleatoria de docentes de ILE, en lugar de una representativa. En total, se recibieron respuestas de unos 79 maestros $(n=79)$. Los participantes son maestros de Inglés, actualmente en servicio en diversas instituciones y programas existentes en República Dominicana.

El cuestionario utilizado constó de 16 preguntas, 15 de las cuales fueron cerradas y una sola abierta. Fue dividido en dos partes: (1) enfocándose en el DI; y (2) enfocándose en el desarrollo docente. Una traducción directa de los ítems de la investigación se encuentra en la sección de apéndices. Referente a la validación del instrumento, los ítems responden a los criterios descritos por Escofet, Folgueiras, Luna y Palou (2016) en los que abogan por enunciados sean claros, precisos y comprensibles, y que se enfoquen en un solo aspecto del estudio, usando un lenguaje claro. El cuestionario se enfocó en presentar llanamente ítems que respondieran a las interrogantes claves de este estudio, por esto no se hay una marcada estratificación de la muestra.

\section{Resultados}

Esta sección presenta los resultados de las encuestas empleadas. Dichos resultados son divididos de acuerdo con las dos preguntas de investigación contempladas en este estudio. Se provee una vista general de las respuestas haciendo hincapié en las preguntas consideradas, por el autor, más reveladoras. Inicialmente, los participantes representaron cierta diversidad en el tiempo activos en la enseñanza de inglés. La tabla 2 lo describe de manera más específica:

Tabla 2. Tiempo de experiencia en la docencia de ILE de los participantes

\begin{tabular}{|c|c|c|}
\hline $\begin{array}{c}\text { Tiempo de } \\
\text { enseñanza }\end{array}$ & $\begin{array}{c}\text { No. de } \\
\text { participantes }\end{array}$ & Porcentaje \\
\hline Menos de 2 años & 22 & 27.8 \\
\hline 2 a 5 años & 18 & 22.8 \\
\hline Más de 5 años & 39 & 49.4 \\
\hline
\end{tabular}

Fuente: elaboración propia. 


\subsection{Diseño instruccional}

Como fue explicado anteriormente, las conceptualizaciones acerca de DI se suscriben a múltiples interpretaciones. El término es ampliamente utilizado en diferentes contextos educativos. En primer lugar, se preguntó a los encuestados referente a su conocimiento general del término "DI". Un $64.6 \%$ de los participantes confirmó no haber escuchado el concepto anteriormente, mientras que solo un $35.4 \%$ supuso saber de qué se trataba. En un esfuerzo por entender la relación del tiempo en servicio con relación al conocimiento en DI, se realizó la siguiente correlación de respuestas. La tabla 3 confirma que los docentes con menos de cinco años de servicio parecen conocer mucho menos de DI en comparación con el grupo de docentes con más de cinco años.

Tabla 3. Conocimiento previo del concepto de "DI" en relación con el grupo de encuestados

\begin{tabular}{|c|c|c|c|c|}
\hline \multicolumn{3}{|c|}{ Grupos de encuestados } & \multicolumn{2}{|c|}{$\begin{array}{l}\text { Conocimiento } \\
\text { del término DI }\end{array}$} \\
\hline \multicolumn{2}{|c|}{$\begin{array}{l}\text { Tiempo de } \\
\text { enseñanza }\end{array}$} & $\begin{array}{l}\text { Porcentaje de } \\
\text { participantes }\end{array}$ & Sí & No \\
\hline 1. & $\begin{array}{c}\text { Menos de } \\
2 \text { ańos }\end{array}$ & 27.8 & $27 \%$ & $73 \%$ \\
\hline 2. & 2 a 5 años & 22.8 & $27 \%$ & $73 . \%$ \\
\hline 3. & $\begin{array}{c}\text { Más de } 5 \\
\text { años }\end{array}$ & 49.4 & $44 \%$ & $56 \%$ \\
\hline \multicolumn{3}{|c|}{ Total de respuestas } & 51 & 28 \\
\hline
\end{tabular}

Fuente: elaboración propia.

Sin embargo, al momento de definir el concepto, las perspectivas de los docentes encuestados variaron significativamente. La tabla 4 presenta las consideraciones de estos docentes con respecto a la definición de DI. La mayoría de los participantes $(31.6 \%)$ piensan que DI se relaciona con las "instrucciones" que preceden una actividad en el aula. El término "instruccional" parece haber guiado esta conclusión. Se entiende que el concepto no es ampliamente discutido en la literatura disponible en español, y su estudio es propio de programas de formación más avanzados relacionados con líneas de desarrollo curricular, y otras áreas.

Tabla 4. Perspectivas de los docentes de ILE con respecto al DI

\begin{tabular}{|l|c|c|}
\hline \multicolumn{1}{|c|}{ Ítems } & Porcentaje & $\begin{array}{c}\text { Total de } \\
\text { Respuestas }\end{array}$ \\
\hline $\begin{array}{l}\text { La forma en la que } \\
\text { organizamos nuestras } \\
\text { instrucciones antes de } \\
\text { una actividad }\end{array}$ & $31.6 \%$ & 25 \\
\hline $\begin{array}{l}\text { La forma en la que se } \\
\text { realiza un currículo/ } \\
\text { sílabo }\end{array}$ & $25.3 \%$ & 20 \\
\hline $\begin{array}{l}\text { Los pasos seguidos por } \\
\text { el docente al momento } \\
\text { de dar instrucciones a } \\
\text { los estudiantes en una } \\
\text { actividad específica }\end{array}$ & $22.8 \%$ & 18 \\
\hline $\begin{array}{l}\text { Los pasos que seguimos } \\
\text { en una clase }\end{array}$ & $20.3 \%$ & 16 \\
\hline
\end{tabular}

Fuente: elaboración propia.

Por otro lado, también se estudiaron los pasos ejecutados por los participantes de manera más específica en sus lecciones. En general, un $64.6 \%$ respondió que la manera correcta de estructurar una lección es "dinámica de inicio, enseñanza, ejemplos, práctica y cierre". Dicha representación de DI concuerda con los resultados de López et al., (2019), en cuyo estudio (aunque en el área de la enseñanza de gramática) se evidenció la relevancia de enfoques lineales de enseñanza en el país. Se observó el siguiente patrón en relación con la experiencia de los docentes participantes. 
Tabla 5. Uso de la Secuencia: "Dinámica de inicio, enseñanza, ejemplos, práctica y cierre”

\begin{tabular}{|c|l|l|}
\hline \multicolumn{2}{|l|}{ Tiempo de enseñanza } & Porcentaje de uso \\
\hline 1. & Menos de 2 años & $83 \%$ \\
\hline 2. & 2 a 5 años & $54 \%$ \\
\hline 3. & Más de 5 años & $60 \%$ \\
\hline
\end{tabular}

Fuente: elaboración propia. Encuesta a docentes de ILE.

En lo adelante, se buscó profundizar en los pasos seguidos por los participantes dentro de esta secuenciación. Debido a la variedad metodológica y a los datos arrojados por la pregunta anterior, solo se consideraron las actividades realizadas en el periodo introductorio y el período de cierre. Con respecto a las introducciones de clases, un 56 \% informó realizar alguna actividad para el inicio de las lecciones. En la figura 1 se explica qué tan frecuentemente los participantes reportaron realizar actividades introductorias en sus lecciones.

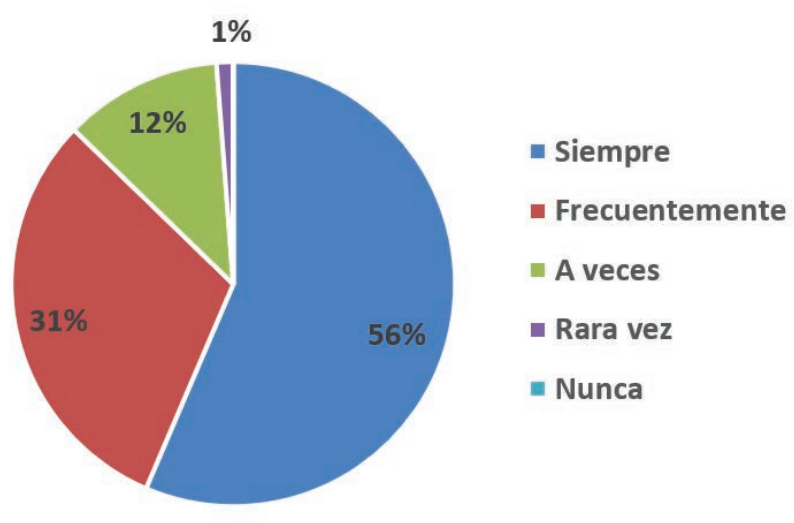

Figura 1. Frecuencia de implementación de actividades de introducción en lecciones de Inglés

Fuente: elaboración propia.

Este gráfico demuestra la presencia de introducciones en las clases de ILE. Pareciera que una mayoría importante de los participantes entienden los beneficios de actividades de apertura dentro de las lecciones. Las actividades utilizadas dentro del área de la enseñanza de idiomas son varias. Dentro de las más utilizadas, de acuerdo con los docentes encuestados, son: (1) inquirir acerca de la clase anterior (26.6 \%), (2) discusión libre acerca de diversos temas (25.3\%), (3) juegos didácticos (15.2\%), discutir una imagen (15.2\%).

Por otro lado, con respecto a las conclusiones, las opiniones parecieron variar más que con respecto a las introducciones de clases. De acuerdo con los resultados, un $48 \%$ de los maestros reportó hacerlas "frecuentemente"; mientras que un $21.5 \%$ dijo realizarlas "a veces". La figura 2 grafica el reporte de frecuencia en que los docentes implementan alguna actividad para esta fase de la experiencia de aprendizaje.

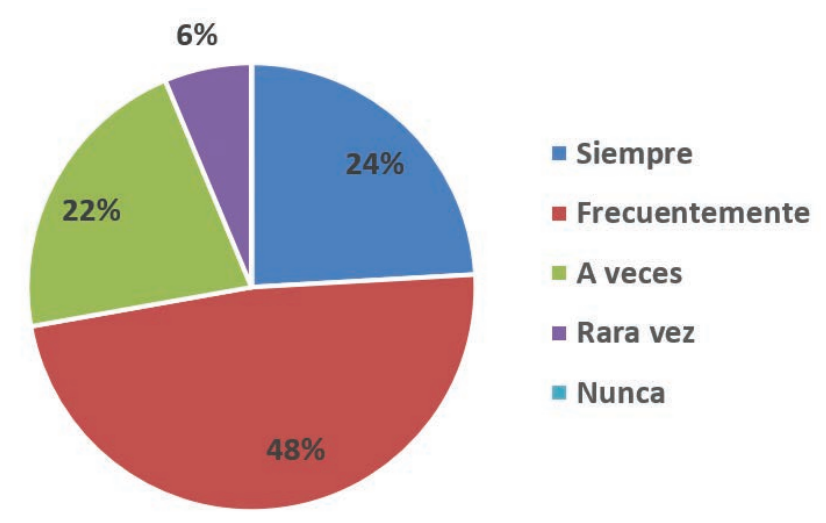

Figura 2. Frecuencia de implementación de actividades de cierre/ conclusión en lecciones de Inglés

Fuente: elaboración propia.

La figura 2 sugiere que los participantes prestan menos atención a la conclusión, en comparación con la frecuencia de implementación de actividades introductorias. Así como en la etapa introductoria, los maestros tienen a su disposición diversas estrategias y actividades para concluir las lecciones. Los resultados arrojaron que la mayoría de los participantes realizan las siguientes actividades para concluir: (1) el uso de preguntas para evaluar aprendizaje (43\%), (2) discusión acerca de lo visto en clase $(20.3 \%)$ y (3) reconstrucción de las actividades llevadas a cabo en el aula (16.5\%), entre otras de menor incidencia.

En general, la información de la encuesta con respecto al DI reveló que los maestros se adhieren a 
enfoques que se observan en la secuencia de la tabla 6: un enfoque lineal del proceso de DI. La información contenida en esta tabla describe los pasos y actividades de manera más específica y a tono con la literatura disponible.

Tabla 6. Diseño instruccional lineal

\begin{tabular}{|c|c|c|}
\hline Introducción & Desarrollo & Conclusión \\
\hline $\begin{array}{l}\text { Actividades lúdicas, } \\
\text { con el propósito } \\
\text { de iniciar la clase } \\
\text { de manera activa } \\
\text { y motivada. } \\
\text { Regularmente } \\
\text { se incluye la } \\
\text { gamificación } \\
\text { del aprendizaje. } \\
\text { Actividades comunes } \\
\text { son: } \\
\text { - Juegos } \\
\text { - Discusiones } \\
\text { - Dinámicas } \\
\text { de grupo e } \\
\text { integración }\end{array}$ & $\begin{array}{l}\text { Enseñanza del punto } \\
\text { céntrico de la lección, } \\
\text { seguida de ejemplos y } \\
\text { práctica. La elección } \\
\text { metodológica del docente } \\
\text { es desplegada en esta } \\
\text { base. } \\
\text { Aquí se observa si la } \\
\text { secuenciación está } \\
\text { basada en el alumno } \\
\text { y su competencia } \\
\text { comunicativa. Ejemplos } \\
\text { de estos enfoques son: } \\
\text { - Enseńanza } \\
\text { deductiva o } \\
\text { inductiva } \\
\text { - Uso de tareas/ } \\
\text { actividades de } \\
\text { precisión gramatical }\end{array}$ & $\begin{array}{l}\text { Una recapitulación } \\
\text { de los procesos y } \\
\text { actividades utilizados } \\
\text { en el aula, guiando } \\
\text { a los estudiantes a } \\
\text { una reflexión de lo } \\
\text { aprendido. Actividades } \\
\text { comunes son: } \\
\text { - Diálogos } \\
\text { - Sesiones de } \\
\text { preguntas y } \\
\text { respuestas } \\
\text { - Llenado de } \\
\text { materiales de } \\
\text { apoyo } \\
\text { - Actividades } \\
\text { de evaluación } \\
\text { formativa } \\
\text { - Discusión acerca } \\
\text { de los objetivos } \\
\text { del día }\end{array}$ \\
\hline
\end{tabular}

Fuente: elaboración propia, basada en el DI encontrado en Richards y Lockhart (1996).

\subsection{Desarrollo profesional docente}

El campo del desarrollo profesional docente es un tema de amplia relevancia para los docentes de lenguas extranjeras. Hay mucho qué hacer para conceptualizar de forma más profunda y expandir la visualización del desarrollo docente en el país. En efecto, un $93.7 \%$ respondió haber conocido o escuchado este término anteriormente. En la siguiente tabla se realiza una correlación del tiempo de enseñanza de los maestros y la forma en la que asocian desarrollo profesional. La información en la tabla 7 resalta cómo la formación profesional parece ser el mayor indicador (84\%) de crecimiento y desarrollo dentro de la carrera docente para los participantes. Solo un $16 \%$ relaciona el crecimiento profesional con la mejora de la práctica docente en el aula.
Tabla 7. Temas asociados al desarrollo profesional en docentes de ILE

\begin{tabular}{|l|l|l|l|l|}
\hline $\begin{array}{l}\text { Grupos de } \\
\text { encuestados }\end{array}$ & \multicolumn{3}{|l|}{ Temas asociados al desarrollo profesional } \\
\hline $\begin{array}{l}\text { Tiempo de } \\
\text { enseñanza }\end{array}$ & $\begin{array}{l}\text { Entrena- } \\
\text { miento, } \\
\text { talleres, } \\
\text { cursos }\end{array}$ & $\begin{array}{l}\text { Promo- } \\
\text { ciones de } \\
\text { puesto }\end{array}$ & $\begin{array}{l}\text { Movilización } \\
\text { de una institu- } \\
\text { ción a otra }\end{array}$ & $\begin{array}{l}\text { Crecer } \\
\text { como } \\
\text { mejor } \\
\text { maestro }\end{array}$ \\
\hline Menos de 2 años & $95 \%$ & $0 \%$ & $0 \%$ & $5 \%$ \\
\hline 2 a 5 ańos & $73 \%$ & $0 \%$ & $0 \%$ & $22 \%$ \\
\hline Más de 5 años & $82 \%$ & $0 \%$ & $0 \%$ & $18 \%$ \\
\hline Total de respuestas & $67=84 \%$ & 0 & 0 & $12=16 \%$ \\
\hline
\end{tabular}

Fuente: elaboración propia.

A partir de estos datos se puede inferir una perspectiva global del desarrollo profesional, que se limita al avance del docente en la institución a la que pertenece, es decir, su promoción a puestos de mayor envergadura como resultado de su tiempo en servicio y preparación.

Dentro de las actividades referentes a desarrollo profesional que han sido realizadas por los maestros, en alguna parte de su carrera, se encuentran las siguientes:

Tabla 8. Actividades orientadas al DPD incluidas en la encuesta

\begin{tabular}{|l|c|}
\hline Actividades & Porcentaje \\
\hline Observación entre pares y proveer retroalimentación & $65.8 \%$ \\
\hline $\begin{array}{l}\text { La revisión de planificaciones de clase a través de un periodo } \\
\text { de tiempo y reflexionar en crecimiento }\end{array}$ & $51.9 \%$ \\
\hline $\begin{array}{l}\text { La participación en cursos de formación (programas en línea, } \\
\text { maestrías, especializaciones, etc.) }\end{array}$ & $48.1 \%$ \\
\hline $\begin{array}{l}\text { La exploración de las creencias del docente con respecto a la } \\
\text { enseñanza, el aprendizaje y el estudiantado }\end{array}$ & $40.5 \%$ \\
\hline El uso de un diario reflexivo & $20.3 \%$ \\
\hline La grabación de videos y su uso para la autoevaluación del docente & $12.7 \%$ \\
\hline
\end{tabular}

Fuente: elaboración propia.

Este resultado sugiere una disposición por parte de los maestros con respecto a su DPD. En el área de la enseñanza de lenguas extranjeras, los programas de DPD permanente escasean. Los maestros demuestran deseo de crecimiento profesional y lo evidencian a través de su reporte de actividades que requieren 
poco involucramiento institucional y pueden ser iniciadas y sostenidas por los docentes mismos. Dentro de tales actividades, la observación entre pares parece ser la más utilizada por los maestros (65.8\%). La investigación educativa sugiere que esta práctica tiene el potencial de fomentar el crecimiento de los maestros envueltos en la misma (Wragg, 1994; 1999; Randall y Thornton, 2001; Richards y Farrell, 2005).

Uno de los focos del desarrollo docente es el futuro de estos en la profesión y su evolución a través del tiempo. La deserción de maestros de la carrera es, a veces, un factor atribuible a la falta de atención al desarrollo docente. La figura 3 explica cómo la mayoría (59.5\%) de los participantes, proyectan continuar en la carrera docente, aparte de su tiempo ya en servicio. La pregunta en cuestión eas: ¿Se ve usted enseñando Inglés en los próximos 10 años?

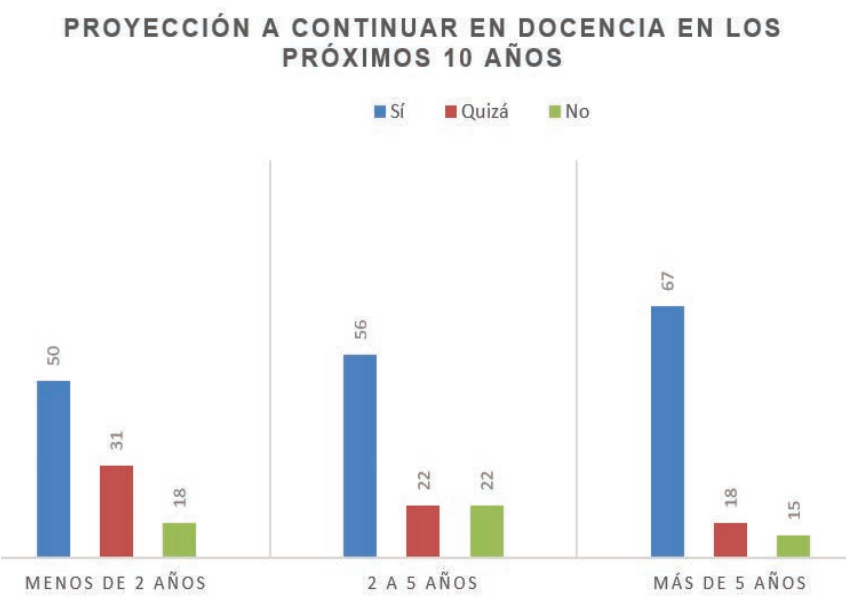

Figura 3. Proyección de continuar en docencia en los próximos 10 años

Fuente: elaboración propia. Los datos representan el porciento de los encuestados.

La figura sugiere que el grupo con mayor tiempo de experiencia docente es el de mayor interés en mantenerse en la carrera docente por los próximos diez ańos. La tendencia parece ser que, a mayor tiempo en docencia, mayor proyección de continuidad en la misma. El grupo con menos de dos años de experiencia presenta mayor nivel de indecisión con respecto a su futuro en la docencia (31\%) en comparación con los demás grupos.

\section{Conclusiones}

Por un lado, el DI representa un reto para los docentes de ILE. La exploración de diversos enfoques de secuenciación en la enseñanza debe proveer a los docentes un entendimiento más holístico del proceso de enseńanza-aprendizaje que les favorezca tomar decisiones instruccionales correctas. Además, expande considerablemente el banco de recursos de los participantes que le permitirán enfrentar de manera efectiva las situaciones y retos que propone el estudiantado. La falta de conocimiento de los docentes de ILE con respecto al "DI" y su confusión de terminología, sugiere un área de mejora en los programas de preparación docente de lenguas extranjeras. Algunos autores suponen una era posmétodo que no se conforma con concepciones fijas e inalterables en el proceso de enseñanza ni en el rol del docente en tal proceso (Kumaravadivelu, 2003); más bien, se concibe un docente hábil y dinámico, capaz de responder de manera diferenciada y relevante a las necesidades que se presentan en el aula. En la estructuración provista por Richard y Lockhart (1996) se sugiere el dinamismo y la flexibilidad del proceso de enseñanza-aprendizaje, facilitando así su comprensión posmétodo. De hecho, estos autores proveen principios que pueden utilizarse en diversos contextos y por docentes de todos los niveles.

Primero, la introducción o apertura en la lección debe orientarse a atraer e involucrar a los estudiantes a la clase en cuestión. Los autores observan que la investigación sugiere que la apertura o entrada a una lección ocupa los primeros cinco minutos de clase y puede tener una influencia en qué tanto pueden los estudiantes aprender de todo el proceso. (Kindsvatter et al.,1988, citado por Richard y Lockhart, 1996). Por otro lado, Marzano (1998) también añade que la especificación y discusión de objetivos en esta etapa es una de las estrategias que guía el establecimiento de un ambiente favorable para la enseńanza y ayuda a construir esquemas mentales que hacen posible el conocimiento (Mercuri y Rea, 2016). De hecho, Richard y Lockhart (1996) abogan por introducciones que sean: 
1. Atractivas en la forma en la que conectan al estudiantado con la nueva experiencia de aprendizaje.

2. Planificadas de tal forma que sean útiles para el docente y todo el proceso de aprendizaje.

3. Orientadas al estudiante.

4. Con un propósito claro.

Segundo, la etapa de desarrollo en las lecciones es el centro del proceso de enseñanza-aprendizaje. En este segmento se discute la nueva información y se guía al estudiante a la nueva experiencia y los nuevos saberes. Aunque los docentes encuestados tienen opiniones diversas con respecto a las introducciones en las lecciones, es indudable el hecho de que sus clases poseen una etapa de desarrollo. Al momento, la literatura en enseńanza de lenguas extranjeras critica duramente enfoques que ven el aprendizaje como un proceso lineal de presentación-práctica-producción. Diversos estudios sugieren que el aprendizaje y adquisición de una lengua extranjera es más complejo que esto, por lo tanto, la enseñanza debe involucrar al estudiante de tal forma que promueva la competencia comunicativa del mismo. En vez de procesos de presentación-práctica, la investigación en el área aboga por: 1) el uso de tareas y 2) el uso de actividades que promuevan la fluidez en los estudiantes.

Van den Branden (2006) explica que una tarea es una actividad en la que una persona se involucra para alcanzar un objetivo que hace necesario el uso del idioma en cuestión. $\mathrm{O}$ sea, que las tareas utilizadas en el aula deben fomentar el uso del inglés en medio de los estudiantes. Estas tareas pueden cumplir con el objetivo de cubrir y usar tanto los puntos gramaticales discutidos como los orientados al vocabulario. Su naturaleza, basada en ejemplos realísticos, permite al docente hacer uso de ellas, con independencia del método comúnmente utilizado. Además, el uso de tareas en un recurso clave para el profesional que se adhiere a una idea situacional o posmétodo para el proceso de enseñanza-aprendizaje. Ejemplos de tareas son: la creación de una lista de compras, la creación de un calendario, la resolución de un problema comunitario, etc. Por otro lado, las actividades con enfoque a la fluidez tienen como enfoque el permitir al estudiante desarrollar competencia comunicativa y perder la timidez al momento de utilizar el idioma. Los maestros dominicanos pueden enriquecer su metodología al utilizar actividades que no tengan un enfoque cerrado, o sea, que su objetivo vaya más allá de corregir al estudiante, sino fomentar la discusión y el compartir de ideas entre todos. Se pueden mencionar como ejemplos de estas actividades las discusiones, las presentaciones informales y los juegos didácticos, entre otras.

Tercero, los resultados del estudio sugieren limitaciones en la conclusión de la lección. Esta etapa parece recibir menor importancia por los maestros. Aunque estos parecen implementar actividades de cierre, se cuestiona el hecho de que estas actividades sigan un principio mayor y estén alineadas con objetivos pedagógicos claros. Acerca de esta etapa, Richard y Lockhart (1996) discuten cómo las actividades de cierre deben tener el propósito de resumir, revisar puntos específicos de la lección, relacionar la lección con la actualidad del estudiante, motivar a los estudiantes por su progreso y reflexionar sobre la utilidad de lo aprendido. Comúnmente, las actividades de cierre son obviadas por los maestros, debido a asuntos de tiempo.

Por otro lado, no solo es importante mirar la secuenciación de la lección en el aula, es de suma importancia, también, ver el progreso del docente, en consideración con su desarrollo profesional. Este estudio sugiere la necesidad de esfuerzos para facilitar el crecimiento del docente de ILE. Es común en los docentes dominicanos entrar en la profesión debido a su dominio de la Lengua, a pesar de no poseer una clara visión de los conceptos y destrezas pedagógicas necesarios para la enseńanza de lenguas extranjeras. No obstante, los docentes dominicanos encuestados muestran interés por su crecimiento. En este caso, la mayoría de los participantes $(57.5 \%$ ) se considera en la carrera docente por diez años más, lo que supone una necesidad de desarrollo profesional latente, que prepare y rete a estos profesionales a innovar y alcanzar los más altos estándares de calidad con respecto a la docencia. Las instituciones de enseñanzas deben ver esta respuesta como favorable para el crecimiento de este campo, y el desarrollo de una República Dominicana bilingüe. Sin embargo, es penoso ver el poco apoyo recibido por los docentes en su desarrollo profesional y la poca practicidad que se le ha da a esta área en la 
enseñanza de Lenguas Extranjeras en el país. De hecho, a sabiendas de esta realidad, las actividades orientadas al desarrollo profesional incluidas en estas encuestas son, en su mayoría, iniciadas y sostenidas por el docente, sin necesidad de una estructura jerárquica.

El DPD debe ser un interés continuo y cardinal de las políticas públicas y privadas en la enseñanza de ILE. Si se espera que nuestra nación avance a la globalización e internacionalización de la educación, es necesario que el docente ocupe su rol como agente imprescindible en este proceso. Las consideraciones hechas por Van Grieken (2018) en pro de una mejora del sistema educativo dominicano, establecen como importantísimas la calidad y la alta competencia desde el proceso de iniciación hasta los procesos internos de evaluación del desempeño del docente dominicano.

Es de gran importancia, también, el conceptualizar de manera más lúcida lo que supone el DPD en el área de Lenguas Extranjeras en el país. El crecimiento profesional no está limitado solamente a la formación del docente a través de programas de inducción o evaluación, sino que es un proceso de auto reflexión y reingeniería de las competencias mismas del docente de cara a su contexto y necesidades. En un intento de estandarizar esta reflexión, es necesario proveer indicadores específicos que sirvan para diagnosticar áreas de fortaleza y oportunidad en la calidad de la enseñanza y del conocimiento docente. Uno de estos esfuerzos es el Marco Docente de Cambridge, el cual es una herramienta útil a ser utilizada para docentes de inglés y para las instituciones. Dicho marco evalúa al docente en las diversas áreas, considerándolo desde los niveles Principiante hasta Experto (Cambridge Assessment English, 2018). Los criterios son los siguientes:

1. El aprendizaje y el estudiante

2. La enseñanza, el aprendizaje y la evaluación

3. Competencia lingüística

4. El conocimiento de idiomas y conciencia

5. Desarrollo profesional y valores

Por cuestiones de espacio, no se definirán los conceptos anteriores. Sin embargo, este es uno de los ejemplos de programas de desarrollo profesional para docentes de ILE que van más allá de la formación continua y que ven al docente como un ente en evolución, de impacto en la realidad educativa nacional.
De manera general, este estudio evidenció la necesidad de experiencias formativas profundas y relevantes para el docente de lenguas extranjeras dominicano. Dichas experiencias deben hacer énfasis en la necesidad de formar docentes que sean críticos de los métodos comunes y que puedan adaptar las lecciones no solo al estudiante, sino a sus necesidades de aprendizaje, de modo que estas sean relevantes y útiles para el alumnado. Queda como reto de las instituciones inmersas en el quehacer educativo el expandir las oportunidades de formación y de fomentar el crecimiento docente a través de la innovación.

En conclusión, este estudio observó una amplia oportunidad de desarrollo, innovación e incorporación de nuevas técnicas de enseñanza y aprendizaje que enriquezcan el DI de las clases de ILE. Dicha diversidad es también atribuible a la formación del docente, la característica del programa y de las percepciones del docente con respecto a la adquisición de lenguas extranjeras. Este estudio aboga por una estructura posmétodo que urja al docente al uso de tareas creativas y comunicacionales, así como a actividades que fomenten la fluidez en el aula.

Por otro lado, el DPD de los maestros de Inglés representa un área de amplia envergadura para la creación de una República Dominicana bilingüe. Para ello, se propone una conceptualización de desarrollo profesional que trascienda los programas de formación inicial y empodere al docente para visualizar su desarrollo desde el inicio de su actividad docente hasta su estado actual. Las instituciones encargadas de la enseñanza de lenguas extranjeras deben priorizar el desarrollo docente como un factor clave para su efectividad e impacto en la sociedad dominicana.

\section{Referencias}

Bonilla, H. (2014). Metodología de enseñanza del idioma Inglés en los liceos: Ercilia Pepín, Américo Lugo, Pedro Francisco Bonó, Pedro Comprés, y los olegios: San Vicente de Paúl y Santa Rosa de Lima, (tesis de maestría). Santo Domingo: Universidad Autónoma de Santo Domingo. Disponible en https://bit.ly/2PcvUdY

Dick, W., \& Carey, L. (1978). The systematic design of instruction (1st ed.). Chicago: Scott: Foresman and Company. 
Escofet, A., Folgueiras, P., Luna, E. \& Palou, B. (2016). Elaboración y validación de un cuestionario para la valoración de proyectos de aprendizaje-servicio. Revista Mexicana de Investigación Educativa, 21(70) 929-949. Disponible en http://bit.ly/2tNV0Yh

Fandiño, Y. (2010), Research as a means of empowering teachers in the 21st century. Educación $y$ Educadores, 13(1), 109-124. Doi: https://doi. org/10.5294/edu.2010.13.1.7

Fandińo-Parra, Y. (2017). Formación y desarrollo docente en lenguas extranjeras: revisión documental de modelos, perspectivas y políticas. Revista Iberoamericana de Educación Superior, 8(22). Doi: https://doi.org/10.22201/iisue. 20072872e.2017.22.232

Gagne, R., Briggs, L., \& Wager, W. (1992). Principles of Instructional Design. Estados Unidos: Hancourt Brace College Publisher. Disponible en https:// bit.ly/2FWkvZV

Gerlach, V., \& Ely, D. (1980). Teaching \& Media: A Systematic Approach. (2da. ed.) Englewood Cliffs: Prentice Hall Inc.

Heinich, R., Molenda, M., Russell, J., \& Smaldino, S. (1999). Instructional media and technologies for learning $\left(6^{\text {th }} E d\right)$. Upper Saddle River, NJ: Merrill: Prentice-Hall.

Hymes, D. (1972). On Communicative Competence. In J.B. Pride J. Holmes (eds) Sociolinguistics. Selected Readings. Penguin, [pp. 269-293]. Disponible en: https://bit.ly/360oXS7

Jardines, F. (2011). Revisión de los principales modelos de diseño instruccional. Innovaciones de Negocios 8(6), 357-389. Disponible en https://bit.ly/ $32 \mathrm{LPlOy}$

Kemp, J. (1985). The Instructional Design Process. New York: Harper \& Grow.

Kumaravadivelu, B. (2003). Beyond methods: Macrostrategies for language teaching. Hartford: Yale University Press.

Larsen, D., \& Anderson, M. (2011). Techniques \& principles in language teaching. New York, NY: Oxford University Press. Disponible en https:// bit.ly/37bzeMJ

López, A. (2019). Spanish Use in the English Classroom: A study of Dominican Students in an Engli-
sh-Only Environment. Beyond Word Journal, 7(1), 20-31. Doi: https://doi.org/10.33508/bw. v7i1.2014

López, A., Martínez, J., Pérez, E., Jiménez, D., \& Mateo, V. (2019). How We Teach Grammar: An Exploratory Study on How Dominican Teachers Deal with Grammar Teaching. MEXTESOL Journal, 43(4), 1-9. Disponible en https://bit. ly/2suM5u4

López, P., \& Fachelli, S. (2015). La encuesta. En P. López-Roldán \& S. Fachelli (Eds.) Metodología de la Investigación Social Cuantitativa. Dipòsit Digital de Documents, Universitat Autònoma de Barcelona. Disponible en: https://bit.ly/ 2 pacADu

Luengo, J. (2004). La educación como objeto de conocimiento. En M. Pozo Andrés, J. Álvarez Castillo, J. Luengo Navas \&, E. Otero Urtza (Eds.). Teorias e instituciones contemporáneas de educación. Disponible en: https://bit.ly/2pN20Ck

Parra, G., \& Leyet, O. (2012). Del diseño instruccional al diseño de aprendizaje con aplicación de las tecnologías. Teoría de la Educación. Educación y Cultura en la Sociedad de la Información, 13(3), 342-360. Disponible en: https://bit.ly/ 2Wcq9hZ

Mato, N. A. (2011). Principlaes métodos de enseñanza de lenguas extranjeras en Alemania. Revista de Lingüistica y Lenguas Aplicadas, (6). Doi: http:// dx.doi.org/10.4995/rlyla.2011.878

Marzano, R. (1998). A theory-based meta-analysis of research on instruction. Disponible en https:// bit.ly/2Jue8yH

Merrill, M.D. (1983). Component Display Theory. En C. Reigeluth (ed.), Instructional Design Theories and Models. Hillsdale, NJ: Erlbaum Associates.

Murray, D., \& Suero, A. (2015). Análisis de las estrategias de enseñanza-aprendizaje utilizadas por los/ las docentes en el análisis de lectura en los niveles intermedio y avanzado de inglés como lengua extranjera de la Escuela de Idiomas (UASD) y el desarrollo de la competencia crítica de los educandos, semestre 2008-2, (tesis de maestría), Universidad Autónoma de Santo Domingo. Disponible en: https://bit.ly/2pMHAtc 
Randall, M., \& B. Thornton (2001) Advising and Supporting Teachers. New York, NY: Cambridge University Press. https://bit.ly/2swJ $5 \mathrm{gV}$

Richards, J., \& Farrell, T. (2005). Professional development for language teachers. New York, NY: Cambridge University Press.

Richards, J., Rodgers, T. (2016). Approaches and methods in language teaching. New York, NY: Cambridge University Press.

Richards, J., \& Lockhart, C. (1996). Reflective teaching in second language classrooms. New York, NY: Cambridge University Press.

Santana, I. (2016). Learning basic English in overcrowded classes for true beginners. Disponible en: https://bit.ly/2N7JpsE
Van den Branden, K. (2006). Task-based language education: From theory to practice. New York, NY: Cambridge University Press.

Van Grieken, C. (2018). Políticas docentes en la República Dominicana avances y desafíos. Ciencia y Educación, 2(2), 13-28. Doi: https://doi.org/ 10.22206/cyed.2018.v2i2.pp13-28

Whitehead, J. (1989). Creating a living educational theory from questions of the kind, how do I improve my practice? Cambridge Journal of Education, 19(1) 41-52. Doi: https://doi. org/10.1080/0305764890190106

Wragg, E. C. (1994) An introduction to classroom observation. Londres, UK: Routledge. 